\title{
Leucemia Linfóide Aguda em Adulto
}

\author{
Acute lymphoid leukemia in adults
}

\section{INTRODUÇÃO}

Apesar dos avanços no tratamento da Leucemia Linfóide Aguda (LLA) na infância e adolescência, tal progresso não tem sido observado na LLA do adulto. Vários protocolos terapêuticos têm sido desenvolvidos. N o entanto, devido à diferença na biologia da doença encontrada entre adultos e crianças, os resultados dos seus tratamentos ainda não se equiparam.

0 índice de remissão completa que, nos adultos, varia entre $65 \%$ e $85 \%$ e a taxa de cura, que aumentou de menos de $20 \%$ para entre $30 \%$ a $40 \%$, ainda permanecem insatisfatórios.

Abaixo estão listados os resultados de alguns protocolos de tratamento da LLA no adulto:

\begin{tabular}{|c|c|c|c|c|c|c|c|c|}
\hline Etudo & Ano & $\begin{array}{c}\text { N-d } \\
\text { Pocienter }\end{array}$ & $\begin{array}{l}\text { Idade } \\
\text { mádsa }\end{array}$ & RC (B) & 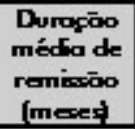 & $\begin{array}{l}\text { Sobreride } \\
\text { Mécfa } \\
\text { (mesel) }\end{array}$ & $\begin{array}{c}\text { Sobrerida Fvre } \\
\text { t doenga } \\
\text { [anod }\end{array}$ & Referência \\
\hline CALGB & 1995 & 197 & 32 & 85 & 29 & 36 & $\begin{array}{l}\text { 46\% (3a) } \\
\text { QT 32\% }\end{array}$ & 1 \\
\hline LALAA 87 & 1993 & 511 & 33 & 76 & 17 & 18 & $\begin{array}{c}\text { AloTMO 43\% } \\
\text { Auto TMO } \\
3 \% \%\end{array}$ & 2 \\
\hline GINEMA & 1992 & 541 & 30 & 84 & 20 & NR & $\begin{array}{l}5 \% \\
(2 a) \\
\end{array}$ & 3 \\
\hline $\begin{array}{r}\mathrm{ECOG} \\
3486\end{array}$ & 1992 & 247 & $35 / 32$ & $73 / 58$ & NR/NR & $9 / 11$ & $\begin{array}{l}17 \% / \\
3 \% \pi \\
(2 a)\end{array}$ & 4 \\
\hline UCSF & 1991 & 109 & 25 & 88 & $N R$ & $N R$ & $\begin{array}{l}35 \pi \\
(5 a)\end{array}$ & 5 \\
\hline MDACC & 1990 & 105 & 30 & 84 & 22 & 19 & $\begin{array}{l}34 \% \\
\text { (5a) }\end{array}$ & 6 \\
\hline SWOG & 1989 & 168 & 28 & 68 & 23 & 18 & NR & 7 \\
\hline $\begin{array}{c}\text { GMAL } \\
83\end{array}$ & 1988 & 368 & 25 & 74 & 24 & 28 & $\begin{array}{c}35 \\
(10 a)\end{array}$ & 8 \\
\hline$\underset{89}{G M A L}$ & 1998 & 41 & 28 & 73 & NR & NR & $\begin{array}{l}4 \text { SF } \\
(8 \mathrm{~g})\end{array}$ & 9 \\
\hline
\end{tabular}

Foi feita a seguinte modificação no protocolo GM ALL-89:

O s estudos sobre resistência a múltiplas drogas têm demonstrado vários mecanismos responsáveis por este fenômeno. Um dado já estabelecido é que existe uma incidência aumentada na expressão desses mecanismos em pacientes adultos, quando comparado a crianças. As antraciclinas são conhecidas como suscetíveis à resistência mediada pela glicoproteína $\mathrm{P}$ (gpP), que é uma glicoproteína transmembrana que confere resistencia cruzada a uma variedade de drogas citotóxicas. Como a idarrubicina é menos suscetível a essa gpP, enquanto a daunorrubicina é mantida na indução, optamos por substituir a doxorrubicina pela idarrubicina na primeira fase da reindução, com o objetivo de atingir células leucêmicas que tenham sobrevivido à indução por este mecanismo de resistência.

\section{AVALIAÇÃO INICIAL}

\section{Avaliação AO dIAGNÓSTICO}

0 paciente ao ser admitido no Serviço de $\mathrm{H}$ ematologia, é avaliado quanto à sua doença de base e presença de doenças associadas que possam interferir na indicação do protocolo terapêutico.

0 critério arbitrário de mais do que $25 \%$ de linfoblastos na medula óssea é usado para distinguir a LLA do Linfoma Linfoblástico. 
O s seguintes exames são, portanto, procedidos:

- Sangue - H emograma e plaquetometria.

- Sangue - TAP e Fibrinogênio.

- Sangue - G licemia.

- Sangue - Avaliação da função renal (uréia e creatinina).

- Sangue - Eletrólitos: Sódio, Potássio, Cloretos, Cálcio e Fósforo.

- Sangue- Transferases(AST Aspartato-aminotransferase e ALT-Alanina-amino-transferase).

- Sangue - Ácido Ú rico.

- Sangue - D esidrogenase Lática (DHL) e

Beta2-M icroglobulina.

- Fezes - Parasitológico.

- Eletrocardiograma.

- Sorologia para: H IV 1 e 2 , H epatite B e C,

HTLV 1 e 2, e sífilis.

- Eletrocardiograma.

- Ecocardiograma (avaliar fração de ejeção).

- Radiografia simples (RX) de tórax, com avaliação do mediastino.

- Punção lombar para avaliação citológica do líquor.

- Cintigrafia óssea e RX do local afetado quando houver dor óssea.

- U Itra-sonografia dos testículos, quando houver aumento testicular.

- Citomorfologia e Citoquímica (PAS, Sudan

Black e Fosfatase Ácida) do sangue periférico ou da medula óssea.

- Imunofenotipagem do sangue periférico ou da medula óssea.

- Citogenética convencional da medula óssea ou do sangue periférico.

- Biologia molecular do sangue periférico ou da medula óssea, servindo, posteriormente, como marcador da doença residual mínima.

FATORES DE MAU PROGNÓSTICO

1. Idade igual ou maior que 60 anos.

2. Leucometria igual ou maior de $30.000 / \mathrm{mm}^{3}$ (na LLA-T a leucometria não é fator prognóstico).

3. Ausência de massa mediastinal na LLA e presença de massa volumosa no Linfoma Linfoblástico.

4. M orfologia L3.

5. Imunofenotipagem $B$ ou $B$ mielóide.

6 . Presença de $t(9: 22)$ ou do gene $B C R / A B L$. 7. Ausência de remissão completa no $D 28$ da Indução.

\begin{tabular}{|c|c|}
\hline Número de futores & $\begin{array}{c}\text { Sobrevida em 3 } \\
\text { anos }\end{array}$ \\
\hline 0 & $97 \%$ \\
\hline 1 & $64 \%$ \\
2 & $49 \%$ \\
\hline 3 & $21 \%$ \\
\hline 4 & $0 \%$ \\
\hline
\end{tabular}

O bs.: pacientes com 0 diagnóstico de LLA de células $B$ são tratados com o protocolo de Linfoma de Burkitt do Serviço de $\mathrm{H}$ ematologia.

PROTOCOLO GMALL- 89

MODIFICADO

\section{Induç̃̃o}

FAse 1:

Prednisona

Vingisina

Daunorrubicina

Daunorrubicina

L-asparaginase

Metrexato

FAsE 2:

Ciclofosamida

Citarabina

6-Mercaptopurina

Metotrexato

Consoidanäio:

Metotrexato

A.jofolínico

L-aparaginase
$60 \mathrm{mg}^{2} / \mathrm{dia}$

$1,5 \mathrm{mg} / \mathrm{m}^{2}$ (máximo $2 \mathrm{mg} /$ dose)

$25 \mathrm{mg} / \mathrm{m}^{2}$

$25 \mathrm{mg} / \mathrm{m}^{2}$ (baixo risco) ou $45 \mathrm{mg} / \mathrm{m}^{2}$ (alto risoo)

$5.000 \mathrm{U} / \mathrm{m}^{2} / \mathrm{dia}$

$12 \mathrm{mg}$

$650 \mathrm{mg} / \mathrm{m}^{2}$ (max $\left.1 \mathrm{~g}\right)$
$75 \mathrm{mg} / \mathrm{m}^{2}$
$60 \mathrm{mg} / \mathrm{m}^{2} / \mathrm{dia}$
$12 \mathrm{mg}$

EV $029,43,57$

EV D $31-34,38-41,45-58$ e 52-55

VO D29 - 57

I D $31,38,45$ e 52
VO D1-28

EV D1, 8,15 e 22

EV D1 e 8

EV D15 e 22

IM D14-28

IT D1

EV D1

EV iníao 36h após início do Metotrexato

M após infusão do Metroxato 
O bs.: pacientes com mais de 50 anos, LLA de baixo risco e LLA-T, a consolidação muda para dois ciclos de:

$\begin{array}{lll}\text { Teniposido } & 60 \mathrm{mg} / \mathrm{m}^{2} / \mathrm{dia} & \text { EV D1- } 4 \\ \text { Citarabina } & 75 \mathrm{mg} / \mathrm{m}^{2} / \mathrm{dia} & \text { EV D1- } 4 \\ \text { Metotrexato } & 12 \mathrm{mg} & \text { IT D1 }\end{array}$

\section{Reinduç̃̃o}

\begin{tabular}{|c|c|c|c|}
\hline \multicolumn{4}{|l|}{ FASE 1: } \\
\hline Dexametasona & $10 \mathrm{mg} / \mathrm{m}^{2} / \mathrm{dia}$ & vo & D1 - 28 \\
\hline Vin crisina & $1,5 \mathrm{mg} / \mathrm{m}^{2}$ (máx $2 \mathrm{mg} /$ dosel) & EV & D1, 8,15 e 22 \\
\hline Idarrubicina & $10 \mathrm{mg} / \mathrm{m}^{2}$ & EV & D1, 8,15 e 22 \\
\hline Metotrexato & $12 \mathrm{mg}$ & IT & D1 \\
\hline \multicolumn{4}{|l|}{ FASE 2: } \\
\hline Ciclofosfamida & $650 \mathrm{mg} / \mathrm{m}^{2}$ (máx $1 \mathrm{~g}$ ) & $\begin{array}{l}\mathrm{EV} \\
\mathrm{FV}\end{array}$ & $\begin{array}{l}\text { D29 } \\
\text { D31-34 } 38-41\end{array}$ \\
\hline 6-Tioguanina & $60 \mathrm{mg} / \mathrm{m}^{2}$ & Vo & $\mathrm{D} 29-42$ \\
\hline
\end{tabular}

\section{Consolidação pós indução (2 ciclos):}

\begin{tabular}{|llll|}
\hline Teniposido & $60 \mathrm{mg} / \mathrm{m}^{2} / \mathrm{dia}$ & EV & D1-4 \\
Citarabina & $75 \mathrm{mg} / \mathrm{m}^{2} / \mathrm{dia}$ & EV & D 1-4 \\
Metotrexato & $12 \mathrm{mg}$ & IT & D1 \\
\hline
\end{tabular}

Radioterapia craniana e em neuro-eixo: sem acometimento inicial - 24G y em 12 sessões; acometimento inicial 30G y em 12 sessões; com massa $30 \mathrm{G}$ y em 12 sessões. N o Linfoma Linfoblástico é feito após a fase 2 da indução, devido à maior chance de recaída em SN C.

\section{MANUTENÇÃO:}

- 6-M ercaptopurina - iniciar com dose baixa até atingir a dose ideal de $60 \mathrm{mg} / \mathrm{m}^{2} /$ dia $\mathrm{VO}$ (ou até a dose tóxica limitante) - durante 18 meses.

- M etotrexato - iniciar com dose baixa até atingir a dose ideal de $20 \mathrm{mg} / \mathrm{m}^{2}$ V0 $1 \mathrm{x} /$ semana (ou até a dose tóxica limitante) durante 18 meses.

\section{EM CASO DE HIPERLEUCOCITOSE INICIAL:}

Em pacientes com grande massa ou leucometria acima ou igual a $30.000 / \mathrm{mm}^{3}$, de modo a evitar a síndrome de lise tumoral, faz-se a citorredução gradativa com prednisona na dose de $30 \mathrm{mg} / \mathrm{m}^{2} /$ dia Vo por 7 dias e vincristina $0,75 \mathrm{mg} / \mathrm{m}^{2}$ (máximo de $2 \mathrm{mg}$ ) EV no primeiro e no sétimo dia da citorredução.

\section{TRANSPLANTE DE MEDULA ÓSSEA ALOGENÉICO}

Pacientes em primeira recidiva ou segunda remissão da $L L A$, pacientes que não obtiveram remissão completa (que mantêm mais do que $5 \%$ de blastos na medula óssea) após a fase de Indução e pacientes com $t(9 ; 22)$ ou bcr-abl (por biologia molecular) têm indicação de transplante alogenéico aparentado (se com até 55 anos de idade) ou não aparentado (se com até 45 anos de idade).

\section{AVALIÇÃO DA REMISSÃO}

No D 15 da fase de Indução, é feito um mielograma para avaliação de remissão medular.

Se o paciente não estiver em remissão medular completa, far-se-á nova avaliação prévia à fase 2 da Indução.

0 mielograma é realizado também a cada mundança de fase do protocolo, assim como na retirada de tratamento.

\section{DURANTE A FASE DE MANUTENÇÃO}

- Sangue: H emograma com plaquetometria, 
Transferases, Ácido Ú rico, U réia e C reatinina

- mensalmente.

- Fezes: Parasitológico a cada 3 meses.

\section{DeteCÇÃO dE FALHA TERAPÊUTICA}

Se ao término da indução não se observa resposta medular completa, o caso deverá ser considerado como de falha terapêutica, devendo ser transferido para protocolo de resgate.

\section{CRITÉRIOS DE RESPOSTA}

Sem resposta: presença de blastos em sangue periférico e mais de $30 \%$ de blastos em medula óssea.

Resposta parcial: normalização do hemograma, porém ainda mais de $5 \%$ e menos de 30\% de blastos em medula óssea.

Resposta completa: H emograma normal e menos de $5 \%$ de blastos na medula óssea.

\section{EXAMES}

\section{AO TÉRMINO DO TRATAMENTO}

- Sangue: H emograma com plaquetometria, Uréia, Creatinina, Ácido Ú rico, D H L e Beta2- M icroglobulina.

- M ielograma.

- Exame citológico do líquor.

\section{APÓS O TÉRMINO DO TRATAMENTO}

O paciente, em resposta completa, deverá ser avaliado a cada 2 meses no primeiro semestre pós-tratamento; a cada 4 meses, no segundo semestre; a cada 6 meses no segundo e terceiro anos; e anualmente a partir do quarto ano fora de tratamento. O s seguintes exames são indicados para essas avaliações:

- Exame físico.

- Exames de sangue: H emograma com plaquetometria, U réia, C reatinina, Ácido Ú rico, DHL e Beta2- M icroglobulina. As sorologias para Sífilis, D oença de Chagas, H epatite B e C, HIV e HTLV deverão ser repetidas no $24^{\circ}$ mês após a última transfusão de hemoderivados.

\section{BIBLIOGRAFIA}

Larson RA, D odge RK, Burns CP, Lee EJ, Stone $R M$, Schulman $P$, et al. A five drug remission induction regimen with intensiveconsolidation for adults with acutelymphoblastic leukemia: $C$ ancer and Leukemia Group B study 8811. Blood 1995;85(8):2025-37.

Boucheix C, D avid B, Sebban C, Racadot E, Bene M C, Bernard A, et al. I mmunophenotype of adult acutelymphoblastic leukemia, clinical parameters and outcomes: an analysis of a prospective trial, including 562 tested patients (LALA87). Blood 1994;84(5):1603-12.

$M$ andelli $F$, Annino L, VegnaM L, CameraA, Ciolli S, D eplano W, et al. GIN EM A ALL 0288: a multicentric study on adult acutelymphoblastic leukemia. Pre liminary results. Leukemia 1992;6(Suppl 2):182-5.

Cassileth PA, Andersen JN , Bennett JM , H oagland HC, M azzaJJ, O 'C onnnell M C, et al. Adult acute lymphocytic leukemia: The Eastern Cooperative Oncology Group experience. Leukemia 1992;6(Suppl 2):178-81.

Linker CA, Levitt LJ, 0 'D onnell M , Forman SJ, RiesCA. Treatment of adult acutelymphoblastic leukemia with intensive cyclical chemotherapy: a follow-up report. Blood 1991;78:2814-22.

Kantarjian H M , WaltersRS, Keating M J, Smith TL, 0 'Brien S, EsteyEH , et al. Results of the vincristine, doxorrubicin and dexametasoneregimen in adults with standard and high-risk acutelymphocytic leukemia. J Clin O ncol 1990;8(6):994-1004.

$H$ ussein KK, D ahlberg S, H ead D, Waddell CC, D abich L, Weich JK, et al. Treatment of acutelymphoblastic leukemia in adults with intensive induction, consolidation and maintenance chemotherapy. Blood 1989;73(1):57-63.

H oelzer $D$, Thiel E, Loffler $H$, BuchnerT, Gauser $A, H$ eil $G$, et al. Prognostic factorsin a multicentric study for treatment of acutelymphoblastic leukemia in adults. Blood 1988; 71(1):123-31.

Ludwing W F, Reider $H$, Bartner $C R, H$ einze $B$, SchuartzS, Gassmann W, et al. Immunophenotipic and genotypic features, clinical characteristics, and treatment outcome of adult pro-B acutelymphoblastic leukemia: results of the G erman M ulticenter Trials GMALL 03/87 and 04/89. Blood 1998;92(6):1898-1909.

Instituto $\mathrm{N}$ acional deC âncer (Brasil). Linfomasna infância e adolescência: condutas do IN CA. Rev Bras C ancerol 2001;47(2):115-23. 